\title{
A MATLAB toolbox for correcting within-individual effects of respiration rate and tidal volume on respiratory sinus arrhythmia during variable breathing
}

\author{
Stefan M. SchulZ \\ University of Würzburg, Würzburg, Germany \\ Erica Ayala \\ Southern Methodist University, Dallas, Texas \\ BERNHARD DAHME \\ University of Hamburg, Hamburg, Germany \\ AND \\ Thomas Ritz \\ Southern Methodist University, Dallas, Texas
}

\begin{abstract}
Respiratory sinus arrhythmia (RSA) is a common estimator of vagal outflow to the heart, dependent on parasympathetic activity. During variable breathing, both respiration rate and tidal volume contribute substantially to within-individual RSA variance. A respiratory control method allows for within-individual correction of the time-domain index of RSA. rsaToolbox is a set of MATLAB programs for scoring respiration-corrected RSA using measurements of cardiac interbeat intervals, respiratory-cycle times, and tidal volumes, recorded at different paced-breathing frequencies. The within-individual regression of RSA divided by tidal volume upon total respiratory cycle time is then used to estimate the baseline vagal tone for each breath of a given total respiratory-cycle time. During a subsequent analysis, the difference between the observed RSA (divided by the tidal volume at each breath) and the RSA divided by the tidal volume that was predicted by the baseline equation serves as an estimate of changes in vagal tone. rsaToolbox includes a graphical user interface for intuitive handling. Modular implementation of the algorithm also allows for flexible integration within other analytic strategies or for batch processing.
\end{abstract}

Obtaining noninvasive measures of autonomic regulation is of great interest to researchers in biological psychology and internal medicine. In this context, respiratory sinus arrhythmia (RSA), or high-frequency heart-rate variability, has frequently been used to estimate vagal outflow to the heart (Berntson et al., 1997; Task Force of the European Society of Cardiology and the North American Society of Pacing and Electrophysiology, 1996). Studies that used pharmacological blockade have clearly demonstrated a dependency of RSA on levels of parasympathetic activity (Katona \& Jih, 1975; Yongue et al., 1982). Additional sources of influence on RSA have been identified, however - most importantly frequency and volume of breathing (e.g., Angelone \& Coulter, 1964; Eckberg, 1983; Grossman, Karemaker, \& Wieling, 1991; Hirsch \& Bishop, 1981). Although the influence of these factors has been known for some time, few studies have attempted to adopt a strategy to account for it (for reviews, see Brown, Beightol, Koh, \& Eckberg, 1993; Grossman \& Taylor, 2007; Ritz \& Dahme, 2006). Control for respiratory confounds in RSA is complicated by the fact that a between-individuals approach to correction (e.g., by analysis of covariance, with tidal volume $\left[\mathrm{V}_{\mathrm{T}}\right]$ and respiration rate $[\mathrm{RR}]$ as covariates) is of limited usefulness because the extent of the influence of these factors varies from individual to individual (Grossman \& Taylor, 2007; Hirsch \& Bishop, 1981; Ritz, Thöns, \& Dahme, 2001).

Following the research of Grossman and colleagues, we have developed a method for within-individual correction of RSA using $\mathrm{V}_{\mathrm{T}}$ and total respiratory cycle time $\left(\mathrm{T}_{\mathrm{TOT}}\right.$, the reciprocal of RR). It is based on observations that RSA normalized by $\mathrm{V}_{\mathrm{T}}$ shows a linear trend across a representative range of $\mathrm{T}_{\text {TOT }}$, and that the changes in $\mathrm{RSA} / \mathrm{V}_{\mathrm{T}}$ at a 


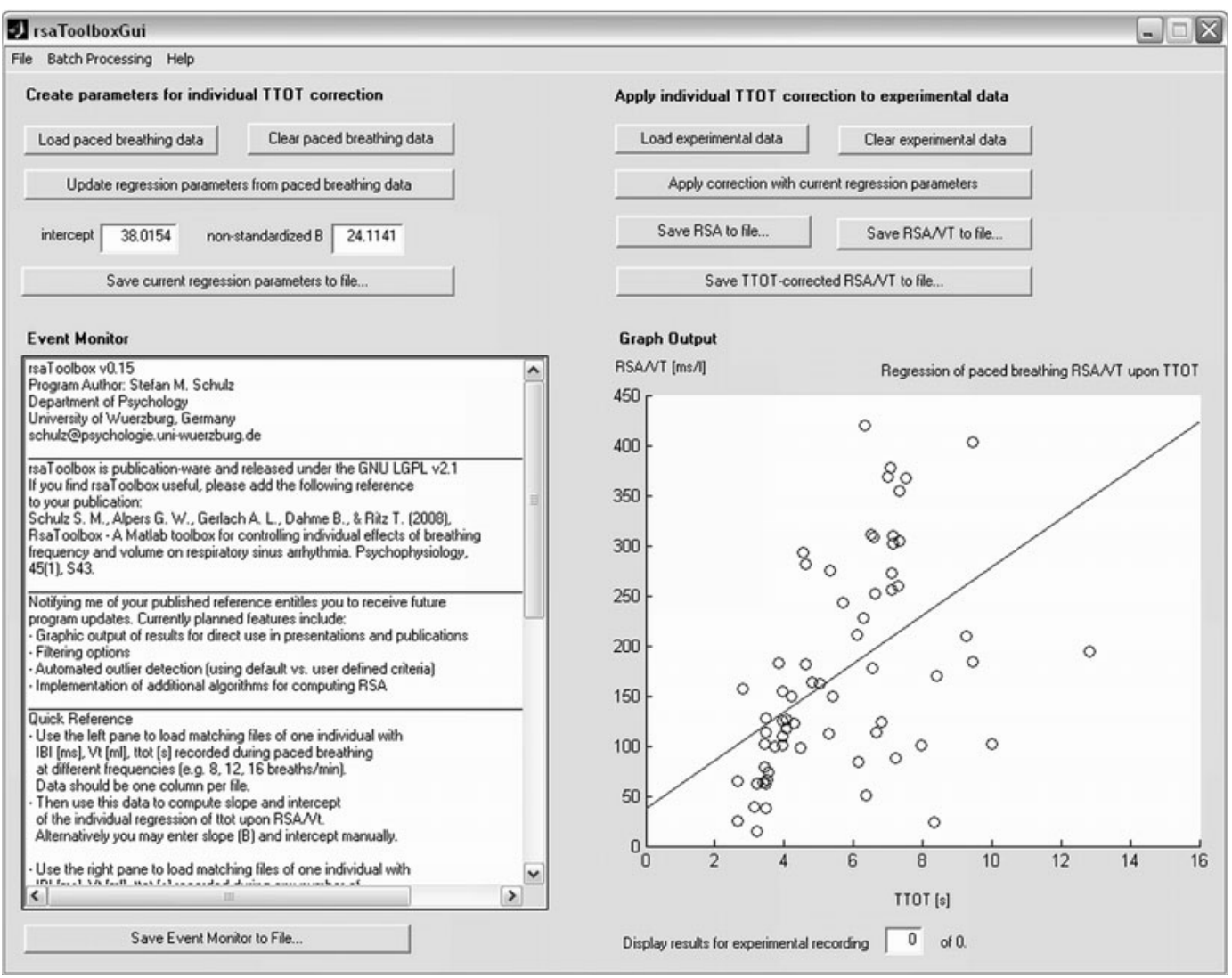

Figure 1. Graphical user interface for rsaToolbox. The left pane allows for stepwise inclusion of total respiratory cycle time ( $\left.\mathrm{T}_{\text {TOT }}\right)$, tidal volume $\left(\mathrm{V}_{\mathrm{T}}\right)$, and interbeat interval (IBI) data of paced breathing recordings to compute individual regression coefficients. Results are presented graphically (as a scatterplot of the regressions). Intercept and nonstandardized $B$ can also be manually entered. The Event Monitor keeps track of user actions and results. The right pane allows for application of the correction to any number of matching $T_{T O T}, V_{T}$, and IBI recordings. Respiratory sinus arrhythmia (RSA), RSA/ $\mathbf{V}_{\mathrm{T}}$, the correction factor, and corrected RSA/ $\mathbf{V}_{\mathrm{T}}$ can be displayed graphically for one recording at a time. These steps may be performed individually or as a whole by clicking on "Batch Processing."

distinct $\mathrm{T}_{\mathrm{TOT}}$ closely track changes in cardiac vagal tone (Grossman \& Kollai, 1993; Saul, Berger, Chen, \& Cohen, 1989). Determining RSA/ $\mathrm{V}_{\mathrm{T}}$ across a representative range of breathing frequencies requires precalibration using at least three or four paced breathing sequences that range from approximately 8 to 16 breaths $/ \mathrm{min}$. The withinindividual regression equation of RSA/ $\mathrm{V}_{\mathrm{T}}$ upon $\mathrm{T}_{\text {TOT }}$ is then used to estimate the baseline vagal tone for each breath of a given $\mathrm{T}_{\mathrm{TOT}}$. For recordings in a subsequent experiment, the difference between the observed RSA/ $\mathrm{V}_{\mathrm{T}}$ at each breath and the RSA/ $\mathrm{V}_{\mathrm{T}}$ that was predicted by the baseline regression coefficients serves as an estimate of vagal tone. It should be noted that the validity of the correction hinges on the intraindividual stability of the relationships between $\mathrm{T}_{\mathrm{TOT}}, \mathrm{V}_{\mathrm{T}}$, and RSA. Previous studies have supported this notion (Ritz, Thöns, \& Dahme, 2001, 2003; Ritz, Wagner, \& Dahme, 1996). Until data on larger samples are available, however, it appears advisable to maximize the intraindividual stability of these associations by keeping settings and tasks during calibration as similar to the experimental conditions as possible. The use of this approach in a number of studies has often yielded easily interpretable findings that differed substantially from those that were based on uncorrected RSA (for a review, see Ritz, 2009).

Measuring respiration-corrected RSA requires measurements of the cardiac interbeat interval (IBI), RR or $\mathrm{T}_{\mathrm{TOT}}$, and $\mathrm{V}_{\mathrm{T}}$. Investigators can follow standard recommendations for guidance on equipment and implementation (Berntson, Quigley, \& Lozano, 2007; Jennings et al., 1981; Lorig, 2007; Ritz et al., 2002).

\section{PROGRAM DESCRIPTION}

We have developed a MATLAB program for the scoring of respiration-corrected RSA. It requires synchronized continuous output of cardiac IBI and of breath-by-breath $\mathrm{T}_{\mathrm{TOT}}$ and $\mathrm{V}_{\mathrm{T}}$.

rsaToolbox comprises a set of MATLAB functions, with a graphical user interface (GUI), for convenient, partially or fully automated correction of individual effects of $\mathrm{T}_{\mathrm{TOT}}$ and $V_{T}$ on RSA. The current version was developed with 
MATLAB 6.5 R13 on an IBM-compatible PC with Windows XP professional OS. rsaToolbox has been tested with MATLAB 6.5 R13 and MATLAB 7.5.0 (R2007b) on an IBM-compatible PC, and with MATLAB 7.6.0 (R2008a) on a Macintosh with OS 10.5 (Intel processor).

Input data for rsaToolbox ( $\mathrm{T}_{\text {TOT }}$ in seconds, $\mathrm{V}_{\mathrm{T}}$ in liters, and IBI in milliseconds) should be saved to commaseparated files $(* . c s v)$, with one data column per file. We recommend filenames with a unique study identifier as a prefix, followed by a counter for the participant number and session. For the different data (i.e., $\mathrm{T}_{\mathrm{TOT}}, \mathrm{V}_{\mathrm{T}}$, and IBI), we recommend extending this prefix with the corresponding parameter abbreviation (e.g., for $\mathrm{T}_{\mathrm{TOT}}$, the filename would be "studyA_p001_ s001_TTOT.csv"). $V_{T}$ must match the corresponding $\mathrm{T}_{\mathrm{TOT}}$ score in each file. Note that the first value in the list of IBIs is used to synchronize the onset of the data vector with the onset of the first breath $\left(\mathrm{T}_{\mathrm{TOT}}\right)$, and it is therefore omitted in the analysis. Furthermore, any value in the list of IBI or $\mathrm{T}_{\text {TOT }}$ may be omitted by flagging the data in additional files (prefix_IBI_flag .csv, and prefix_TTOT_flag.csv, respectively). This procedure can be used to omit irregular breaths, ectopic heartbeats, or other sources of irregular data from the analysis. For convenience, an appropriate data example is provided with the rsaToolbox, including an example of how to flag irregular data.

The following provides a description of typical usage and optional features for rsaToolbox, as well as a feature overview of the program functions.

rsaToolbox.m opens a convenient interactive GUI for invoking all the necessary steps of the algorithm, both stepwise and in different automatic batch modes. The GUI requires the following files: rsaToolboxGui.m, rsaToolboxGuiHelp.m, rsaToolboxGuiInfo.m, rsaToolboxGui.fig, rsaToolboxGuiHelp.fig, and rsaToolboxGuiInfo.fig. Entering "rsaToolbox" at the MATLAB prompt starts the program. A quick reference (shown in the Event Monitor; see Figure 1) explains the main functions. Additional details can be accessed by opening rsaToolbox Help in the menu of the GUI. All operations are logged and can be saved to a text file. The results are displayed graphically and may be saved to comma-separated files (see below).

rsaToolbox uses the following set of functions, implementing the generic algorithm that was reported in Ritz and Dahme (2006).
Both rsaLoad.m and rsaBatchLoad.m invoke the operating system's standard interface for importing the required data. In batch mode, the results are always saved in the folder in which the input file was opened.

rsaPeakValley. $m$ detects the shortest and the longest heart period ( $\mathrm{IBI}_{\min }$ and $\mathrm{IBI}_{\max }$, respectively) within each breathing cycle. The (uncorrected) peak-valley RSA for each breathing cycle is then computed as the difference between the two $\left(\mathrm{IBI}_{\max }-\mathrm{IBI}_{\min }\right)$ under the following conditions: (1) $\mathrm{IBI}_{\min }$ precedes $\mathrm{IBI}_{\max }$ in time, and (2) both IBIs are not constant within one breathing cycle. For rare cases in which more than one IBI with equal length is detected within one breathing cycle (the probability of this event increases with lower sampling rates), there are two options: (1) The last $I I_{\min }$ must fall before the first IBI $I_{\max }$, or (2) the first $\mathrm{IBI}_{\min }$ must fall before the last $\mathrm{IBI}_{\max }$ - with the latter, most lenient, option as the default setting.

rsaClearFlag. $m$ cleans the data according to the criteria that are specified in rsaPeakValley.m. RSA scores that do not meet the conditions that are mentioned above are deleted in the calibration data set. These scores are set to zero when raw RSA is computed or when the correction is applied to experimental data (see Grossman \& Wientjes, 1986).

rsaNormalizeByVT.m computes RSA normalized by $\mathrm{V}_{\mathrm{T}}$ by dividing $\mathrm{IBI}_{\max }-\mathrm{IBI}_{\min }$ for each breathing cycle by the respective $\mathrm{V}_{\mathrm{T}}$.

rsaRegress.m provides a calculation of the withinindividual regression of RSA/ $\mathrm{V}_{\mathrm{T}}$ upon $\mathrm{T}_{\mathrm{TOT}}$ across the whole paced-breathing task (i.e., all paced-breathing epochs from the baseline calibration procedure concatenated). This requires installation of MATLAB's statistics toolbox, "stats" (www.mathworks.com/products/ statistics/). In addition, or as an alternative, it is possible to output RSA/ $\mathrm{V}_{\mathrm{T}}$ (variable "tempRVCol") and $\mathrm{T}_{\mathrm{TOT}}$ (variable "tempTTOTCumCalCol") in a tab-delimited text file (DataForExternalRegression_RSA_upon_VT_and_TTOT .txt) for external computation of the regression using a statistics program of your choice.

rsaApplyCal.m then uses the regression coefficients intercept and $B$, the least squares estimator of the slope, from the concatenated paced-breathing trials to compute $y^{\prime}$, which is the RSA/ $\mathrm{V}_{\mathrm{T}}$ value that is predicted on the basis of the preexperimental paced-breathing trials. Intercept and slope may also be entered manually from an external regression computation. Next, corrected RSA indices for respiratory cycles in the experiment data $\left(y_{\text {residual }}=\right.$ corrected RSA $/ \mathrm{V}_{\mathrm{T}}$ ) can be computed as the

Table 1

Effects of Posture on Components That Are Relevant to Respiratory Sinus Arrythmia: Interbeat Interval (IBI), Tidal Volume $\left(V_{T}\right)$, and Total Respiratory-Cycle Time $\left(\mathbf{T}_{\mathrm{TOT}}\right)$

\begin{tabular}{|c|c|c|c|c|c|c|c|c|c|c|c|c|}
\hline & \multicolumn{6}{|c|}{ Participant 1} & \multicolumn{6}{|c|}{ Participant 2} \\
\hline & \multicolumn{2}{|c|}{ IBI (msec) } & \multicolumn{2}{|c|}{$\mathrm{V}_{\mathrm{T}}(\mathrm{L})$} & \multicolumn{2}{|c|}{$\mathrm{T}_{\mathrm{TOT}}(\mathrm{sec})$} & \multicolumn{2}{|c|}{$\underline{\mathrm{IBI}(\mathrm{msec})}$} & \multicolumn{2}{|c|}{$\mathrm{V}_{\mathrm{T}}(\mathrm{L})$} & \multicolumn{2}{|c|}{$\mathrm{T}_{\mathrm{TOT}}(\mathrm{sec})$} \\
\hline & $M$ & $S D$ & $M$ & $S D$ & $M$ & $S D$ & $M$ & $S D$ & $M$ & $S D$ & $M$ & $S D$ \\
\hline Quiet sitting & 1,006 & 66 & 0.145 & 0.022 & 3.348 & 0.471 & 713 & 72 & 1.328 & 0.181 & 9.266 & 1.124 \\
\hline Standing & 706 & 80 & 0.235 & 0.213 & 3.929 & 1.207 & 691 & 88 & 1.68 & 0.221 & 9.813 & 0.765 \\
\hline Supine-eyes open & 1,102 & 77 & 0.133 & 0.015 & 3.397 & 0.936 & 824 & 88 & 1.15 & 0.183 & 8.634 & 0.646 \\
\hline Supine-eyes closed & 1,137 & 98 & 0.21 & 0.299 & 10.638 & 12.958 & 847 & 69 & 0.855 & 0.311 & 7.483 & 1.493 \\
\hline
\end{tabular}




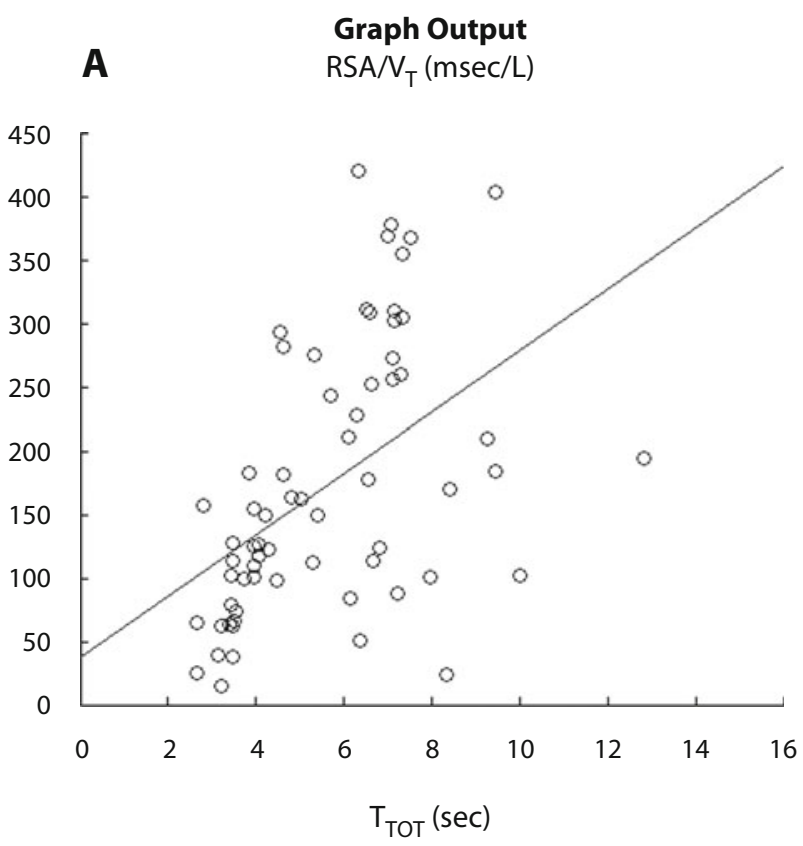

Regression of Paced Breathing RSA/ $\mathrm{V}_{\mathrm{T}}$ Upon $\mathrm{T}_{\mathrm{TOT}}$
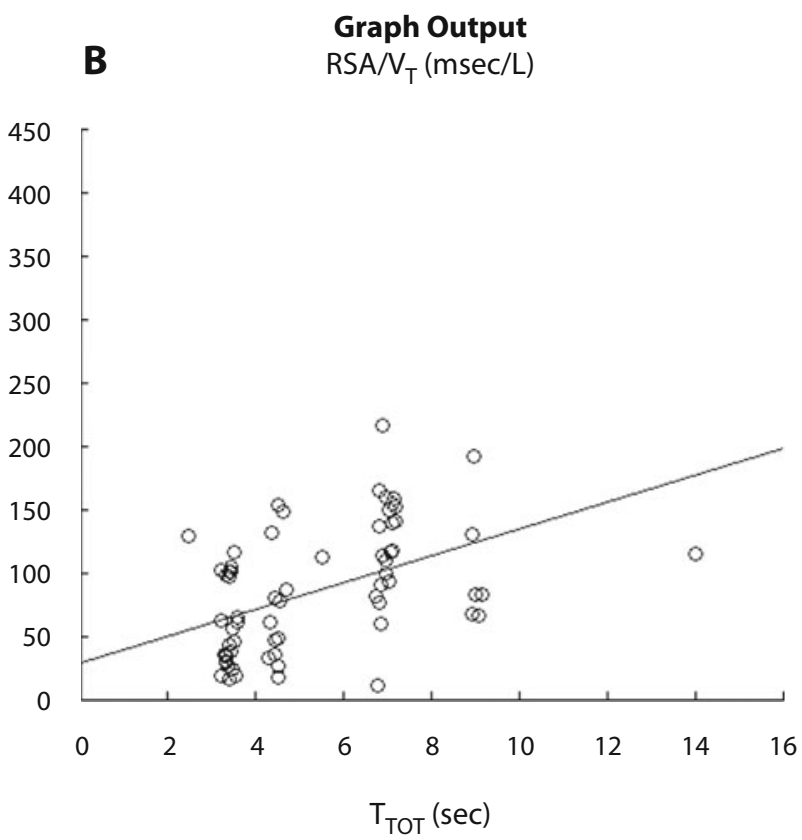

Regression of Paced Breathing RSA/ $\mathrm{V}_{\mathrm{T}}$ Upon $\mathrm{T}_{\text {TOT }}$ deviation from the value predicted on the basis of the preexperimental paced-breathing trials: $y_{\text {residual }}=y-y^{\prime}$ $\left(y=\right.$ experiment RSA $/ \mathrm{V}_{\mathrm{T}}$, and $y^{\prime}=$ predicted RSA $\left./ \mathrm{V}_{\mathrm{T}}\right)$.

Additionally, the corrected RSA index for data is computed by applying a correction for distortions that were caused by nonlinearity, which is found with extremely long breathing cycles (breathing cycles exceeding a 10-sec duration are set to $10 \mathrm{sec}$ as a default).

There are three additional routines: rsaAppendLine.m is used to add data vectors of variable length to an existing data array, equalizing different length with $\mathrm{NaN}$ (i.e., MATLAB notation for "Not a Number"); rsaStrmult.m duplicates a string variable a set number of times; and rsaTostr.m converts integers to strings with a variable number of digits, adding leading zeros if necessary.

Data output may be saved manually via standard file dialogues. In batch mode, output is generated automatically in the folder of the corresponding input files. The prefix in the resulting file list is then replaced by the file name of the corresponding input file: prefix_RSA.csv contains the simple RSA; prefix_RSAbyVT.csv contains RSA corrected for effects of $\mathrm{V}_{\mathrm{T}}$; prefix_TTOTcorrected RSAbyVT.csv contains the corrected RSA index as the deviation from the value that was predicted on the basis of the calibration recordings; and prefix_TTOTcorrected RSAbyVT_cutoff.csv contains the same index, but with an applied cutoff criterion for nonlinearity at extreme values. In addition, slope, intercept, $R^{2}, d f$, and $p$ of the regression that is computed for paced-breathing data may be saved to prefix_regressionParameters.csv. Figure 1 shows the GUI.

\section{EXAMPLE DATA}

The data in the following example were drawn from a study on cardiac vagal outflow across posture variations. We expect the following rank order for cardiac vagal activity in the examined conditions: supine eyes closed $>$ supine eyes open $>$ quiet sitting $>$ standing.

The RSA correction is demonstrated using data from 2 participants who differed in the nature and extent to which their RSA was influenced by paced-breathing frequency, $\mathrm{T}_{\mathrm{TOT}}$, and $\mathrm{V}_{\mathrm{T}}$. Both participants were women (age, 27 vs. 31 years; height, 163 vs. $165 \mathrm{~cm}$; weight, 55 vs. $62 \mathrm{~kg}$ ). The preexperimental paced breathing consisted of three 3-min blocks of breathing at $8,10.5$, and $18 \mathrm{cpm}$, with respective $\mathrm{T}_{\mathrm{TOT}} \mathrm{S}$ of 7.5, 5.7, and $3.3 \mathrm{sec}$. In following the auditory pacing signal, participants usually do not

Table 2

Figure 2. (A) Regression graph of the within-individual regression of respiratory sinus arrhythmia divided by tidal volume $\left(\operatorname{RSA} / V_{T}\right)$ upon total respiratory-cycle time $\left(\mathbf{T}_{\text {TOT }}\right)$, used to estimate baseline vagal tone for each breath of a given total respiratory-cycle time for Participant 1 . Note that the slope for Participant 1 is more than twice as steep as that for Participant 2. (B) Regression graph of the within-individual regression of RSA/ $V_{T}$ upon $T_{\text {Tот }}$, used to estimate baseline vagal tone for each breath of a given total respiratory-cycle time for Participant 2.
Regression Parameters Indicating Different Effects of Respiratory Cycle Time on Respiratory Sinus Arrythmia Normalized (i.e., Divided) by Tidal Volume for Two Individuals

\begin{tabular}{lccccc}
\hline & Intercept & Slope & $R^{2}$ & $d f$ & $p$ \\
\hline Participant 1 & 38.015 & 24.114 & .236 & 63 & $<.001$ \\
Participant 2 & 29.259 & 10.529 & .226 & 66 & $<.001$ \\
\hline
\end{tabular}




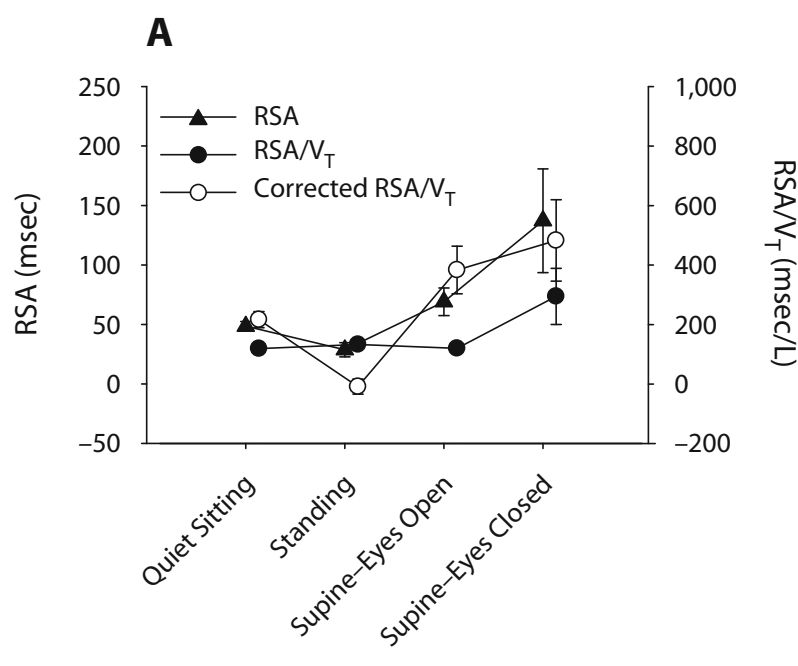

B

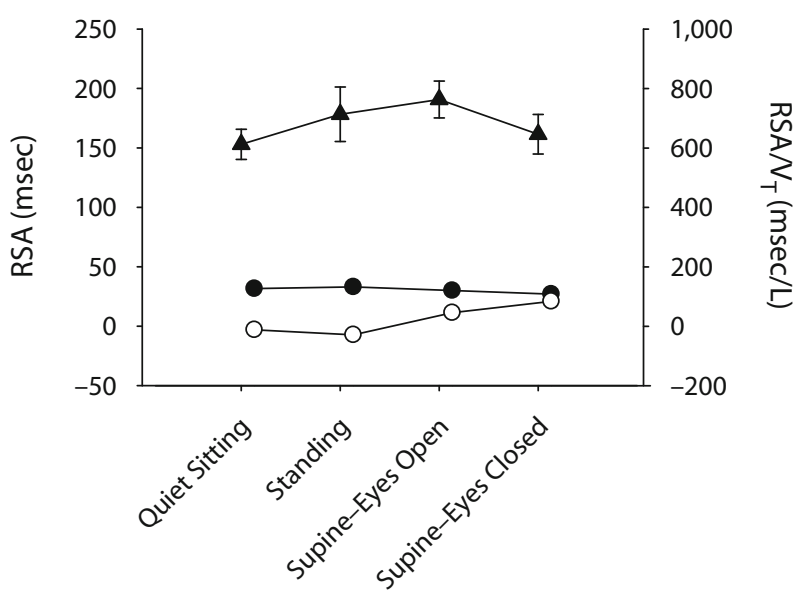

Figure 3. (A) Comparison of correction procedures for respiratory sinus arrhythmia (RSA) of Participant 1: first, when only tidal volume $\left(V_{T}\right)$ is taken into account; second, when $V_{T}$ and individual differences of total respiratory-cycle time $\left(T_{T O T}\right)$ on $R S A / V_{T}$ are taken into account. Note that corrected RSA/ $\mathbf{V}_{T}$ shows the best fit to theoretically predicted changes of vagal tone. (B) Comparison of correction procedures for RSA of Participant 2: first, when only $V_{T}$ is taken into account; second, when $V_{T}$ and individual differences of $T_{T O T}$ on RSA/ $V_{T}$ are taken into account. Note the smaller effect of the individual correction for effects of $\mathbf{T}_{\text {TOт }}$ when compared with Participant 1.

match these breathing frequencies exactly, showing slight variations in the measured $\mathrm{T}_{\mathrm{TOT}} \mathrm{S}$ around the target value.

Table 1 provides means of the relevant RSA parameters, IBI, $\mathrm{V}_{\mathrm{T}}$, and $\mathrm{T}_{\mathrm{TOT}}$ for the two example participants. Participant 1 showed rather low mean heart rates (53$59 \mathrm{bpm})$, except during standing (84 bpm); slightly higher than normal RR ( $15-18$ breaths $/ \mathrm{min}$ ), except in the supine eyes-closed condition ( 6 breaths/min); and generally very low mean tidal volumes $(0.133-0.235 \mathrm{~L})$ across postures. Participant 2 showed generally higher mean heart rates in the sitting $(84 \mathrm{bpm})$ and standing $(87 \mathrm{bpm})$ postures and slightly smaller mean heart rates in both supine postures (eyes open, 73 bpm; eyes closed, 71 bpm). Mean RRs were generally lower and less variable between conditions $(6-8 \mathrm{cpm})$ in this participant, and $\mathrm{V}_{\mathrm{T}} \mathrm{s}$ were 4 - to 10fold greater than in Participant 1 . The 2 participants thus showed nearly reverse patterns in levels of $R R$ and $V_{T}$.

Figures $2 \mathrm{~A}$ and $2 \mathrm{~B}$ show the within-individual regression of RSA/ $\mathrm{V}_{\mathrm{T}}$ upon $\mathrm{T}_{\mathrm{TOT}}$ for both participants from the preexperimental paced-breathing episodes, with regression coefficients and variance explained listed in Table 2. Participants did not differ in intercepts and variance explained $\left(R^{2}\right)$, but they did differ in the slope of the regression, with the slope for Participant 1 being more than twice as steep as that for Participant 2.

These intercepts and slopes were then applied to calculate RSA over corrected $\mathrm{V}_{\mathrm{T}}$ for the experimental data. Within-individual means of the uncorrected RSA, RSA/ $\mathrm{V}_{\mathrm{T}}$, and RSA $/ \mathrm{V}_{\mathrm{T}}$ corrected for $\mathrm{T}_{\mathrm{TOT}}$ are shown in Figures $3 \mathrm{~A}$ and $3 \mathrm{~B}$. Note that the unit of the uncorrected RSA is milliseconds, displayed on the left ordinate, whereas the unit for RSA $/ \mathrm{V}_{\mathrm{T}}$ and corrected $\mathrm{RSA} / \mathrm{V}_{\mathrm{T}}$ is milliseconds per liter, shown on the right ordinate.

Comparisons with the original uncorrected RSA thus had to be restricted to the rank orders of the withinindividual means in the four situations. For Participant 1, the expectations were best met by corrected $\mathrm{RSA} / \mathrm{V}_{\mathrm{T}}$, although the uncorrected RSA also showed the expected rank order. Uncorrected RSA suggested that there was only a slight reduction of cardiac vagal activity from quiet sitting to standing posture. The within-individual means of $\mathrm{RSA} / \mathrm{V}_{\mathrm{T}}$ showed a rather flat trend, only poorly discriminating between the four conditions. In Participant 2, the expected rank order was again reflected best by corrected $\mathrm{RSA} / \mathrm{V}_{\mathrm{T}}$. Uncorrected RSA differed from the expectation, with higher values in the standing posture than in the quiet sitting posture, and with higher values in the supine eyesopen condition than in the supine eyes-closed condition. It should be noted that paced-breathing precalibrations in this study were implemented in only one posture (sitting). It is possible that individual precalibrations at each posture could improve the precision in differentiating cardiac vagal activity between postures.

\section{CONCLUSION}

The data from both participants show that correcting RSA for $\mathrm{V}_{\mathrm{T}}$ and $\mathrm{T}_{\mathrm{TOT}}$ (or RR) does contribute to the sensitivity in detecting predicted changes in cardiac vagal activity. Performing such corrections is not a cumbersome obligation, therefore, but rather helps to elucidate expected cardiac vagal output in a more distinctive and valid manner. The program that is available for these calculations is userfriendly and provides convenient access to state-of-the-art RSA correction procedures. We hope that this program will increase understanding of individual differences in the relationship between cardiac vagal functioning and respiration, aiding researchers in implementing a more sensitive and valid alternative to interpreting uncorrected RSA.

\section{AUTHOR NOTE}

This work was supported in part by a scholarship from the Gottlieb Daimler- and Karl Benz-Foundation to S.M.S. The first author thanks 
Alexander Gerlach, University of Münster, for his guidance in learning MATLAB, and Paul Pauli and Georg W. Alpers, University of Würzburg, for their continuous support. Correspondence concerning this article should be addressed to S. M. Schulz, Department of Psychology, University of Würzburg, Marcusstrasse 9-11, D-97070 Würzburg, Germany (e-mail: schulz@psychologie.uni-wuerzburg.de).

\section{REFERENCES}

Angelone, A., \& Coulter, N. A., JR. (1964). Respiratory sinus arrhythmia: A frequency dependent phenomenon. Journal of Applied Physiology, 19, 479-482.

Berntson, G. G., Bigger, J. T., Jr., Eckberg, D. L., Grossman, P., KaufmanN, P. G., MaliK, M., ET AL. (1997). Heart rate variability: Origins, methods, and interpretive caveats. Psychophysiology, 34, 623648

Berntson, G. G., Quigley, K. S., \& Lozano, D. (2007). Cardiovascular psychophysiology. In J. T. Cacioppo, L. G. Tassinary, \& G. G. Berntson (Eds.), Handbook of psychophysiology (3rd ed., pp. 182-210). New York: Cambridge University Press.

Brown, T. E., BeIGHTOL, L. A., KoH, J., \& ECKBERG, D. L. (1993). Important influence of respiration on human $\mathrm{R}-\mathrm{R}$ interval power spectra is largely ignored. Journal of Applied Physiology, 75, 2310-2317.

ECKBERG, D. L. (1983). Human sinus arrhythmia as an index of vagal cardiac outflow. Journal of Applied Physiology, 54, 961-966.

Grossman, P., Karemaker, J., \& Wieling, W. (1991). Prediction of tonic parasympathetic cardiac control using respiratory sinus arrhythmia: The need for respiratory control. Psychophysiology, 28, 201-216.

Grossman, P., \& Kollai, M. (1993). Respiratory sinus arrhythmia, cardiac vagal tone, and respiration: Within- and between-individual relations. Psychophysiology, 30, 486-495.

Grossman, P., \& TAYLOR, E. W. (2007). Toward understanding respiratory sinus arrhythmia: Relations to cardiac vagal tone, evolution and biobehavioral functions. Biological Psychology, 74, 263-285.

Grossman, P., \& WientJes, K. (1986). Respiratory sinus arrhythmia and parasympathetic cardiac control: Some basic issues concerning quantification, applications and implications. In P. Grossman, K. H. L. Janssen, \& D. Vaitl (Eds.), Cardiorespiratory and cardiosomatic psychophysiology (pp. 117-138). New York: Plenum Press.

HiRSCH, J. A., \& BISHOP, B. (1981). Respiratory sinus arrhythmia in humans: How breathing pattern modulates heart rate. American Journal of Physiology, 241, H620-H629.
Jennings, J. R., Berg, W. K., Hutcheson, J. S., Obrist, P., Porges, S., \& Turpin, G. (1981). Publication guidelines for heart rate studies in man. Psychophysiology, 18, 226-231.

Katona, P. G., \& JiH, F. (1975). Respiratory sinus arrhythmia: Noninvasive measure of parasympathetic cardiac control. Journal of Applied Physiology, 39, 801-805.

LoRIG, T. S. (2007). The respiratory system. In J. T. Cacioppo, L. G. Tassinary, \& G. G. Berntson (Eds.), Handbook of psychophysiology (3rd ed., pp. 231-243). New York: Cambridge University Press.

RITZ, T. (2009). Studying noninvasive indices of vagal control: The need for respiratory control and the problem of target specificity. Biological Psychology, 80, 158-168.

Ritz, T., \& Dahme, B. (2006). Implementation and interpretation of respiration sinus arrhythmia measures in psychosomatic medicine: Practice against better evidence? Psychosomatic Medicine, 68, 617-627.

Ritz, T., Dahme, B., DuBois, A. B., Folgering, H., Fritz, G. K., HarVER, A., ET AL. (2002). Guidelines for mechanical lung function measurements in psychophysiology. Psychophysiology, 39, 546-567.

Ritz, T., Thöns, M., \& DAhme, B. (2001). Modulation of respiratory sinus arrhythmia by respiration rate and volume: Stability across posture and volume variations. Psychophysiology, 38, 858-862.

Ritz, T., ThÖNS, M., \& DAнme, B. (2003). Ventilation-correction of respiratory sinus arrhythmia: The sensitivity of the respiratory modulation of vagal outflow is stable across time [Abstract]. Psychophysiology, 40, $\mathrm{S} 71$.

Ritz, T., Wagner, C., \& Dahme, B. (1996). A paced breathing procedure for the inter- and intraindividual estimation of cardiac vagal tone [Abstract]. Psychophysiology, 33, S70.

Saul, J. P., Berger, R. D., Chen, M. H., \& Cohen, R. J. (1989). Transfer function analysis of autonomic regulation: II. Respiratory sinus arrhythmia. American Journal of Physiology, 256, H153-H161.

Task Force of the European Society of Cardiology and the North American Society of Pacing and Electrophysiology (1996). Heart rate variability: Standards of measurement, physiological interpretation, and clinical use. European Heart Journal, 17, 354-381.

Yongue, B. G., McCabe, P. M., Porges, S. W., Rivera, M., Kelley, S. L., \& ACKLES, P. K. (1982). The effects of pharmacological manipulations that influence vagal control of the heart on heart period, heart-period variability and respiration in rats. Psychophysiology, 19, 426-432.

\section{APPENDIX}

To obtain the latest version of rsaToolbox, please contact S. M. Schulz (schulz@psychologie.uni-wuerzburg de). rsaToolbox is distributed under the GNU Lesser General Public License (LGPL), Version 2.1, February 1999, published by the Free Software Foundation. This makes rsaToolbox essentially free software; however, if you like rsaToolbox, please express your recognition of the time and dedication that was invested to make this tool available by citing this article in your publications.

Please check the readme.txt that is included with rsaToolbox for details on verifying the integrity of the software, installation, further optional usage, and the most recent updates or bug fixes. 